\title{
Design and Implement Controllable Multicast Based Audio/Video Collaboration
}

\author{
Xuan Zhang ${ }^{1,2}$, Dongtao Liu ${ }^{1}$, and Xing $\mathrm{Li}^{1,2}$ \\ ${ }^{1}$ Network Research Center \\ ${ }^{2}$ Department of Electronic Engineering \\ Tsinghua University ,Beijing China, 100084 \\ zhangx@cernet.edu.cn, ldt@mails.tsinghua.edu.cn, \\ xing@cernet .edu.cn
}

\begin{abstract}
Multicast based audio/video collaboration system is one of representative applications in next generation internet. Adopting multicast technique could save bandwidth for multipoint-to-multipoint audio/video communication. Lack of ubiquitous native multicast limits the application, and current multicast based $\mathrm{A} / \mathrm{V}$ collaboration systems lack effective control mechanism. This paper introduces one controllable audio/video collaboration system based on multicast. The control and management on audio/video collaboration are presented. The system has been implemented and applied on CERNET.
\end{abstract}

Keywords: Controllable collaboration, audio/video, multicast.

\section{Introduction}

The multipoint audio/video collaboration systems have played important roles in Next Generation Internet [1][2]. Among collaboration techniques IP multicast has advantage on saving bandwidth for group communication. This makes it advanced comparing to centralized system on large scale multi-party A/V collaboration.

Some IP multicast based A/V collaboration applications Access-grid[3] INDIVIA[4], have been employed in internet. But problems remain to be solved to these current systems. The control and manage on current multicast-based $\mathrm{A} / \mathrm{V}$ collaboration systems are not effective and not easy to be employed when the scale is large, for example, the voice echo and noise problem during collaboration.

This paper introduces the IP multicast based A/V collaboration system. The control and management on multicast based audio/video tools are presented, performance monitor and congestion control are described. We have implemented and applied the controllable audio/video collaboration system on CERNET successfully.

\section{Control and Management on Audio/Video Collaboration}

In multicast based audio/video collaboration system, each user could receive all video and audio streaming from other users in the group. Users talk to each other equally, there are no centralized control or manage. This equality in many-to-many advances 
interactive quality for collaboration. On the contrary, lack of control and management cause problems during collaboration such as noise or echo. How to focus current speaker's video among the dazzling video windows of the group is another issue.

To manage and control the participants' audio/video in collaboration, we propose one mechanism based on chairman control panel to control and manage the $\mathrm{A} / \mathrm{V}$ during collaboration. The control panel is managed by chairman. During session course the control panel could monitor the audio/video states and control video/audio by sending control message. The messages are sent via unicast or multicast way according to request.

The multicast based audio/video tools we adopted are originally from rat/vic [5].We modified the tools to meet the goal of management and control.

\subsection{Control and Manage on Audio}

In audio collaboration, users in group could speak equally and freely. Avoiding the unwanted voice such as noise or echo is hoped. Chairman or administrator should be able to control it when abnormal voices happened. Here we adopt the scheme combining voice volume monitoring and remote control as figure 1.

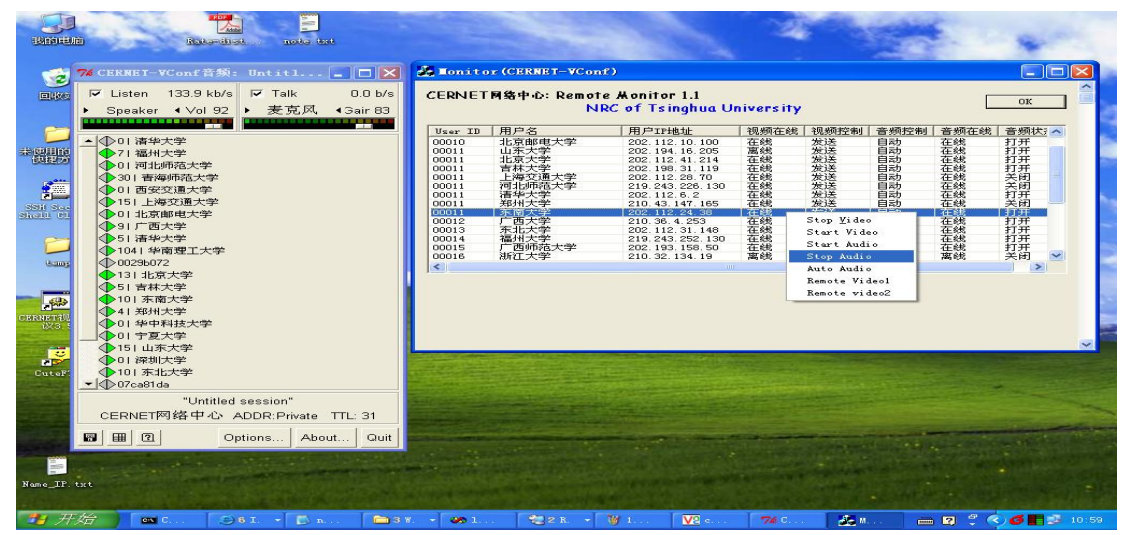

Fig. 1. The chairman control panel with audio monitor interface

The left of figure 1 presents the audio monitor interface, the users' voice volume values ranging from 0 to 16383 are displayed in user-list. We call the voice volume as powermeter. According to powermeter chairman could judge which user is speaking or making noise. When any speaker makes noise or echo, chairman could mute the speaker remotely by sending Audio/mute message to the speaker from control panel. Figure3 shows how chairman mutes user (202.112.24.38) remotely via control panel.

\subsection{Control and Management on Video}

During multicast based video collaboration, users could receive all users' video streaming and see all the others' video windows simultaneously. When the number of video windows is large, how to focus the current speaker video window is necessary. 
Commonly, the current speakers, chairman and local video should be focused and their video windows should be enlarged.

Figure 2 shows one layout for video windows, the main speakers, local video and slider window are enlarged, the other participants' video remain as stamp windows.

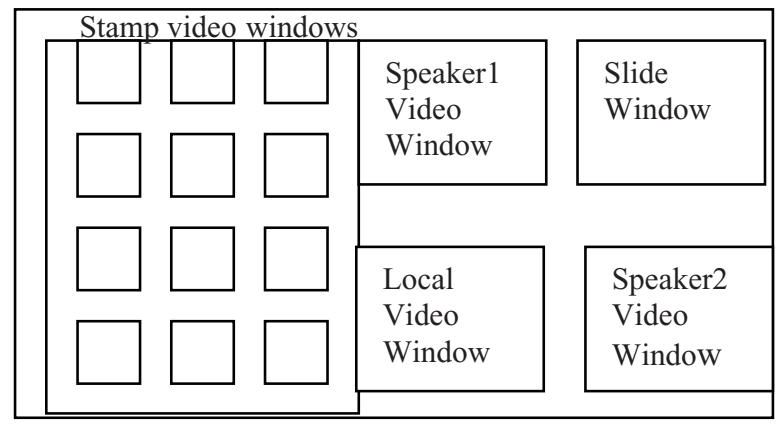

Fig. 2. Video window layout during collaboration

How to build and maintain the focusing-based video layout automatically for all users is important for group collaboration. Typically, when the current speakers change, the enlarged video windows for speakers should be changed accordingly to all users synchronously.

We implement the video layout by defining four types of Enlarged Video Windows (EVW) class: EVW/local, EVW/slide, EVW/speakerl and EVW/speaker2. The EVW classes are identified by video source identification SSRC. Among them EVW/local and $E V W /$ slide are usually constant and can be designated at beginning.

But EVW/speakerl and EVW/speaker2 would change during collaboration course. We dlefine messages Video/speakerl and Video/speaker2 (as table 1) as EVW control message for EVW/speakerl and EVW/speaker2. When one new speaker begins to talk, the chairman could select the new speaker from the user list in control panel (as figure 1), and send the current speaker's SSRC to all users by sending Video/speakerl and Video/speaker2 message. When end user receives the newly Video/speaker messages, the end system would change their current speaker EVW automatically according to received control message. The Video/speakerl and Video/speaker2 messages are multicast to all users in the group, so all the users could switch their speakers' EVW synchronously. Figure 3 shows one instance of video layout with EVW and stamp video, during one forum on IPv6.

Table 1. Control messages for video

\begin{tabular}{ll}
\hline Message type & Description \\
\hline Video/bit-rate-send & bit rate of video sending for flow control \\
Video/speaker1 & EVW control message for $E V W /$ speaker1 with SSRC \\
Video/speaker2 & EVW control message for $E V W /$ speaker2 with SSRC \\
\hline
\end{tabular}




\section{Application Cases}

The multicast based audio/video collaboration systems have been employed to more than 38 cities covering all provincial capitals of China. In 2006, some checking and report meetings for research project were held via $\mathrm{A} / \mathrm{V}$ collaboration system. Figure 3 shows one scene on IPv6 forum via the collaboration system on CERNET.

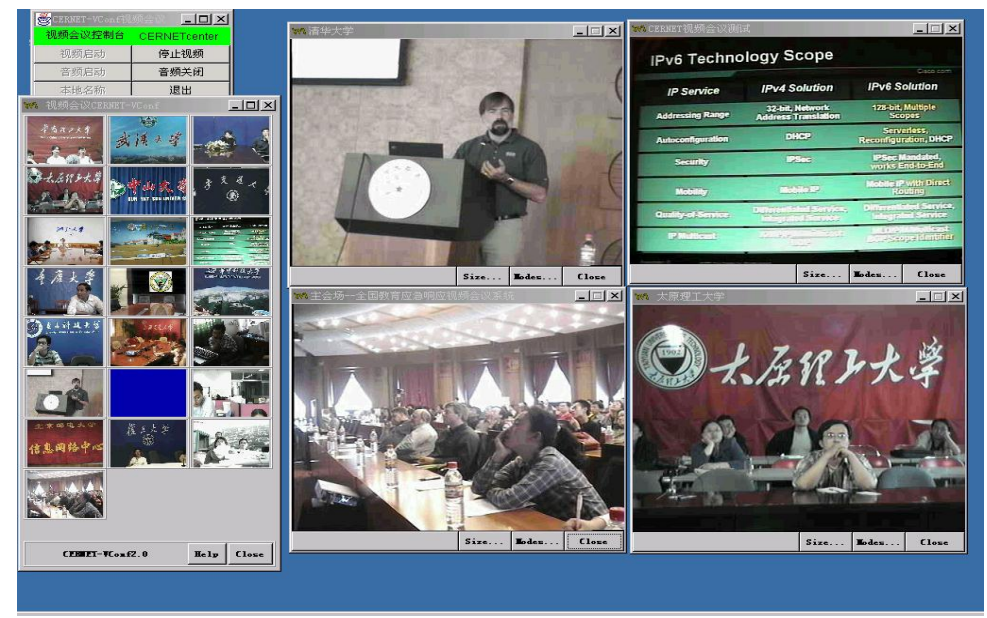

Fig. 3. Instance of video layout with EVW, one application case on IPv6 forum

\section{Conclusion}

The controlling and managing are important for many-to-many A/V collaboration. In this paper, we introduce one controllable multicast based audio/video collaboration systems. The audio/video collaboration controlling and managing mechanisms are discussed. We have implement and applied the collaboration systems on CERNET. The application would extend to CERNET2 (the Chinese next generation internet).

\section{References}

1. Internet 2 consortium, http://internet2.edu/ .

2. Geoffrey Fox, Wenjun $\mathrm{Wu}$, Ahmet Uyar, Hasan Bulut,Shrideep Pallickara, "Global Multimedia Collaboration System", 1st International Workshop on Middleware forGrid Computing, Rio de Janeiro, Brazil, (June 2003)

3. Access Grid Project, http://www.accessgrid.org

4. W.T. Ooi, P. Pletcher, and L.A. Rowe,INDIVA: Middleware for Managing a Distributed Media Environment, SPIE Multimedia Computing and Networking, (January 2004).

5. http://www-mice.cs.ucl.ac.uk/multimedia/software/ 